\title{
Identification of Urinary Exosomal miRNAs for the Non-Invasive Diagnosis of Prostate Cancer
}

This article was published in the following Dove Press journal:

Cancer Management and Research

\author{
Zhuo $\mathrm{Li}^{1-3, *}$ \\ La-Xiu $\mathrm{Li}^{3,4, *}$ \\ Yan-Jun Diao (iD ${ }^{3}$ \\ Juan Wang ${ }^{3}$ \\ Yun $\mathrm{Ye}^{1,3}$ \\ Xiao-Ke $\mathrm{Hao}^{2,3,5}$
}

'Department of Laboratory Medicine, The First Affiliated Hospital of X'an Medical University, Xi'an, Shaanxi 710077, People's Republic of China; ${ }^{2}$ The National Engineering Research Center for Miniaturized Detection Systems, College of Life Science, Northwest University, Xi'an, Shaanxi 710069, People's Republic of China; ${ }^{3}$ Department of Laboratory Medicine, Xijing Hospital, The Fourth Military Medical University, Xi'an, Shaanxi 710032, People's Republic of China; ${ }^{4}$ Department of Laboratory Medicine, Xi'an Fourth Hospital, Xi'an, Shaanxi 710004, People's Republic of China; ${ }^{5}$ School of Medicine, Northwest University, Xi'an, Shaanxi 710069. People's Republic of China

*These authors contributed equally to this work
Correspondence: Xiao-Ke Hao School of Medicine, Northwest University, Xi'an, Shaanxi 710069, People's Republic of China Email haoxkg@I26.com
Background: Novel and non-invasive biomarkers with higher sensitivity and specificity for the diagnosis of prostate cancer (PCa) is urgently needed. In this study, we used nextgeneration sequencing (NGS) to characterize the genome-wide exosomal miRNA expression profiling in urine specimens and explored the diagnostic potential of urinary exosomal miRNAs for PCa.

Methods: Urinary exosomal microRNA expression profiling was performed by nextgeneration sequencing (NGS) and then validated by quantitative real-time PCR.

Results: Significant downregulation of urinary exosomal miR-375 was observed in PCa patients compared with healthy controls, while the expression levels of urinary exosomal miR-451a, miR-486-3p and miR-486-5p were found to be significantly up-regulated in the PCa patients. Furthermore, the expression level of urinary exosomal miR-375 showed a significant correlation with the clinical T-stage and bone metastasis of patients with $\mathrm{PCa}$ $(P<0.05)$. Receiver operator characteristic curve demonstrated that the urinary exosomal miR-375, miR-451a, miR-486-3p and miR-486-5p levels can be used to differentiate PCa patients from healthy controls, with area under the curves (AUCs) of 0.788, 0.757, 0.704 and 0.796 , respectively. The urinary exosomal miR-375 was found to be superior in discriminating between localized and metastatic PCa with an AUC of 0.806. Moreover, PCa patients can be distinguished from patients with benign prostatic hyperplasia by using a panel combining urinary exosomal miR-375 and miR-451a with an AUC of 0.726.

Conclusion: These findings demonstrate that the urinary exosomal miRNAs can serve as novel and non-invasive biomarkers for diagnosing and predicting the progression of $\mathrm{PCa}$.

Keywords: prostate cancer, next-generation sequencing, urinary, exosomal miRNAs

\section{Background}

Prostate cancer $(\mathrm{PCa})$ is one of the most common malignant tumours in the male urinary system. ${ }^{1}$ Although the conventional biopsy is the gold standard for the diagnosis of this tumour, its application is limited due to its invasive procedure. Currently, prostate-specific antigen (PSA) is the most commonly used biomarker for prostate cancer screening. Serum PSA testing for the early detection of PCa, however, strongly depends on the patient's age and the local prevalence of the disease. $^{2}$ Most importantly, there are other factors in addition to $\mathrm{PCa}$ that can increase the PSA level, such as the presence of benign prostatic hyperplasia (BPH), infection and chronic inflammation, ${ }^{3}$ leading to a significant overdiagnosis and subsequent overtreatment of indolent PCa. The PSA level, Gleason score, and radiological features are difficult to predict the progression of PCa. Therefore, novel and non-invasive biomarkers with high sensitivity and specificity 
for the diagnosis of $\mathrm{PCa}$ are urgently needed. Exosomal microRNAs (miRNAs or miRs) in circulating body fluids have recently been reported to augment the diagnosis and management of various diseases, including cancer. ${ }^{4-7}$

Exosomes are small membrane-bound vesicles ranging in size from 30 to $100 \mathrm{~nm}$, which are secreted by numerous cell types into the extracellular environment and can be found in biological fluids such as blood, semen, saliva and urine. ${ }^{8-10}$ Exosomes contain proteins and various types of RNA molecules including miRNAs. It has been reported that miRNA-rich exosomes can be taken up by neighbouring or distant cells, and subsequently modulate those recipient cells. ${ }^{11}$ Recent research has revealed that exosomal miRNA profiling can reflect its cell of origin. ${ }^{12,13}$ In particular, the exosomal miRNAs derived from urine have been identified in recent years. ${ }^{14,15}$ Recently, extensive attention has been focused on using exosomal miRNAs as a source of biomarkers, because their contents resemble those of the cell of origin. A substantial number of exosomal miRNAs have been reported as diagnostic, prognostic, or even therapeutic biomarkers in cancer patients. ${ }^{16-20}$ Therefore, exosomal miRNAs may serve as valuable non-invasive biomarkers for the diagnosis and prognosis of certain diseases.

Over the last two decades, researchers have emphasized the importance of aberrant miRNA expression, which is a relevant driving force in the initiation and progression of PCa. However, there are few studies reported on the use of exosomal miRNAs in urine specimens for diagnosis and prognosis in patients with $\mathrm{PCa}$. In this study, to explore the diagnostic value of urinary exosomal miRNA for prostate cancer, next-generation sequencing (NGS) technology was used to characterize the genome-wide exosomal miRNA expression profile in urine from PCa patients and healthy controls, and quantitative real-time PCR (qRT-PCR) assay was then performed to further validate the candidate miRNAs. The flow chart of the experimental design was shown in Supplemental Figure S1.

\section{Patients and Methods}

\section{Patients and Sample Collection}

All urine samples were obtained from the Xijing Hospital of The Fourth Military Medical University (Xi'an, People's Republic of China). All the patients with PCa or $\mathrm{BPH}$ disease enrolled in this study were newly diagnosed cases (no prior treatment). The diagnosis of $\mathrm{PCa}$ was confirmed by histological examination or prostate biopsy. Histopathologic Gleason scoring of the tumours was performed according to the WHO criteria. Tumours were staged according to the seventh edition of the American Joint Committee on Cancer tumour-node-metastasis (TNM) system. Clinical T-classification of $\mathrm{PCa}$ was divided as organ-confined (T1/T2) or non-organ-confined (T3/T4). First-catch morning urine samples $(15-40 \mathrm{~mL})$ were obtained from all participants without prostate rectal exam or massage, and then centrifuged at $10,000 \times \mathrm{g}$ for 30 mins to remove cell debris and sediment. They were subsequently collected in $50 \mathrm{~mL}$ Eppendorf conical tubes and stored at $-80^{\circ} \mathrm{C}$ before exosomal RNA isolation. In this study, six PCa patients and three healthy controls were used as the NGS discovery cohort. Subsequently, another $47 \mathrm{PCa}$ patients, $29 \mathrm{BPH}$ patients and 25 age- and gendermatched healthy individuals were used as the qRT-PCR validation cohort (Table 1). This study was approved by the Medical Ethics Committee of the Xijing Hospital of The Fourth Military Medical University (No. XJYYLL$2,015,129)$ and was performed in accordance with the regulatory guidelines regarding clinical assays. Informed consent was obtained from all participants or their legal guardians in this non-invasive study. This study was conducted in accordance with the Declaration of Helsinki.

\section{Urinary Exosomes Isolation}

Exosomes were isolated from urine samples using ExoQuick-TC for tissue culture media and urine (System Biosciences, Mountain View, CA, USA) according to the manufacturer's protocol. In brief, the urine samples were thawed on ice. Subsequently, $2 \mathrm{~mL}$ of ExoQuick-TC reagent was added to $10 \mathrm{~mL}$ of urine, and the samples were

Table I Expression of seven Significantly Deregulated miRNAs Between Localized PCa Patients and Metastatic PCa Patients Determined by NGS Technology

\begin{tabular}{|l|l|l|l|l|}
\hline \multirow{2}{*}{ miRNAs } & \multicolumn{2}{|l|}{ Expression Level } & $\begin{array}{l}\text { Up/ } \\
\text { Down }\end{array}$ & $\begin{array}{l}\mid \text { log2 } \\
\text { (FC)| }\end{array}$ \\
\cline { 2 - 3 } & $\begin{array}{l}\text { Localized } \\
\text { PCa }\end{array}$ & $\begin{array}{l}\text { Metastatic } \\
\text { PCa }\end{array}$ & & \\
\hline hsa-miR-16-2-3p & 22.45 & 0.00 & Down & 3.67 \\
hsa-miR-375 & 67.18 & 5.28 & Down & 17.78 \\
hsa-miR-45 Ia & 2039.60 & 70.16 & Down & 4.86 \\
hsa-miR-486-3p & 1288.70 & 27.18 & Down & 5.92 \\
hsa-miR-486-5p & 4096.00 & 67.68 & Down & 5.57 \\
hsa-miR-3I82 & 20.00 & 1.18 & Down & 4.09 \\
hsa-miR-36I5 & 18.33 & 0.00 & Down & 17.48 \\
\hline
\end{tabular}


thoroughly mixed by inverting three times. They were then incubated at $4^{\circ} \mathrm{C}$ for $12 \mathrm{~h}$, followed by centrifuging at $1500 \times \mathrm{g}$ for $30 \mathrm{mins}$. The supernatant was discarded, and the pellets were collected for RNA isolation. The presence of exosomes in the pellets was confirmed by transmission electron microscopy (Supplemental Figure S2).

\section{Transmission Electron Microscopy (TEM)}

An aliquot of the exosomal pellet was resuspended in phosphate-buffered saline, and a drop of the suspension was placed on a sheet of parafilm. A copper grid was placed on the drop for $2 \mathrm{~min}$ at room temperature and was subsequently placed onto a drop of $2 \%$ phosphotungstic acid for a $2 \mathrm{~min}$ staining process. The grid was air-dried for several minutes and was examined by using a Tecnai G2 Spirit electron microscopy (FEI Co., Hillsboro, OR, USA).

\section{Exosomal RNA Isolation}

Exosomes were extracted from equal amounts of urine samples collected from healthy controls, $\mathrm{BPH}$ and $\mathrm{PCa}$ patients. RNA was isolated from the exosomes using miRNeasy Serum/Plasma kit (Qiagen, Hilden, Germany) according to the manufacturer's protocol. To regulate the variability of exosomal miRNA expression levels, at the beginning of RNA isolation, $2 \mu \mathrm{L}$ of cel-miR-39 (RiboBio, Guangzhou, China) was added to each sample as a spike-in control. ${ }^{21-23}$

\section{Sequencing Library Preparation and Sequencing Data Analysis}

To select miRNAs whose expression levels were altered in PCa patients compared with healthy controls, exosomal microRNA profiling of nine urine samples (6 $\mathrm{PCa}$ patients including 3 localized and 3 metastatic $\mathrm{PCa}$ patients and 3 age-matched healthy controls) was performed using Illumina NGS technology. Small RNA libraries were constructed using RNA extracted from exosomes. Index libraries were pooled equally for sequencing (Illumina HiSeq2500 platform). The exosomal miRNA RNA-seq data were processed primarily by miRBase 21 , which indicated miRNA types and expression levels. Exosomal miRNA profiling was normalized using read counts per million mappable miRNA sequences.

\section{Validation of Exosomal miRNAs Using qRT-PCR}

The qRT-PCR was performed using a PrimeScript RT Reagent kit and a SYBR Premix Ex Taq kit (Takara Bio, Inc., Shiga,
Japan) according to the manufacturer's protocols. Briefly, $2 \mu \mathrm{L}$ of total RNA from each sample was reverse transcribed using a PrimeScript RT Reagent kit and specific stem-loop primers that were purchased from RiboBio. The qPCR reactions were performed on an ABI 7500 Fast Detection system (Applied Biosystems, Foster City, CA, USA) using an SYBR Premix Ex Taq kit and sequence-specific primers purchased from RiboBio. Each experiment was carried out in triplicate, and the mean value of the three-cycle threshold was used for further analysis. Cel-miR-39 was used to normalize the exosomal miRNA expression analysis, and the relative quantification analysis of exosomal miRNA was calculated using the $2^{-\Delta \Delta \mathrm{Ct}}$ method. ${ }^{24}$ Exosomal miRNAs with threshold cycle $(\mathrm{Ct})$ values $>35$ were excluded from the analysis.

\section{Statistical Analysis}

Statistical analysis was performed using the MannWhitney $U$-test on log-transformed data between the groups. The box plots represent miRNA levels relative to cel-miR-39, transformed into quantities using the formula $2^{-\Delta \Delta \mathrm{Ct}}$. The box plots and correlation analysis were performed using GraphPad Prism 5. Abnormally distributed data are presented as the median (IQR). $P$ value $<0.05$ was considered statistically significant.

Receiver operator characteristic (ROC) curves were constructed using SPSS 16.0 to estimate the feasibility of using the specific exosomal miRNAs to differentiate between the $\mathrm{PCa}$ patients and the healthy controls. Meanwhile, the area under the curve (AUC) was used to test the power of using the specific exosomal miRNAs and PSA to distinguish PCa patients from $\mathrm{BPH}$ patients or to differentiate between localized PCa and metastatic PCa. In brief, the logistic regression was first carried out using miRNA expression levels, and the predicted probabilities were calculated for each single miRNA or all miRNAs together. The AUC was calculated with $95 \%$ confidence intervals.

\section{Results \\ Expression Profiling of Urinary Exosomal miRNAs in Prostate Cancer and the Selection of Candidate Exosomal miRNAs for Validation}

Morphological confirmation of exosomes isolated from urine samples was evaluated by TEM (See Supplemental Figure S1). Evaluating aberrant urinary exosomal miRNA expression in $\mathrm{PCa}$ can provide a promising tool to identify a specific biomarker for the diagnosis and prediction of 
PCa. In the initial screening phase using NGS technology, urine samples were collected from six PCa patients (3 localized PCa patients and 3 metastatic PCa patients) and three healthy controls. The standardized count of sequencing reads (total sequencing tags in the library) could be used to compare miRNA expression levels between PCa patients and healthy controls. A genome-wide expression profiling of exosomal miRNAs obtained by Illumina NGS technology showed that urine from PCa patients had 39 down-regulated miRNAs and 14 up-regulated miRNAs compared with urine from the healthy controls (Table 2), and urine from metastatic PCa patients had 7 downregulated miRNAs compared with the urine of localized PCa patients (Table 1). For validation, four significantly up-regulated miRNAs (miR-16-2-3p, miR-451a, miR-486$3 p$ and miR-486-5p) and one down-regulated miRNA (miR-375) were selected, which showed a mean foldchange $>2$ between $\mathrm{PCa}$ patients and healthy controls, as well as between localized PCa and metastatic PCa.

\section{Validation by qRT-PCR}

To further validate the sequencing data, the five differentially expressed urine exosomal miRNAs were examined by using qRT-PCR in a validation sample cohort composed of $47 \mathrm{PCa}$ patients and 25 healthy controls. The clinical and demographic characteristics of the PCa patients are summarized in Table 1. qRT-PCR results demonstrated that the expression of miR-375 was significantly downregulated in urine samples of $\mathrm{PCa}$ patients compared with those of healthy controls (Figure 1A). In contrast, the expression levels of miR-451a, miR-486-3p and miR-486-5p were significantly upregulated in PCa samples compared with healthy controls (Figure 1C-E). More importantly, the trend of the alteration of the expression of the four miRNAs was generally concordant between the sequencing data and the validation cohort. In addition, miR16-2-3p displayed a differential expression tendency between NGS and qRT-PCR, but there was no significant difference in the relative expression of miR-16-2-3p in PCa samples compared with samples from healthy controls (Figure 1B). Therefore, miR-16-2-3p was excluded from further study. This phase generated a panel of four miRNAs (miR-375, miR-451a, miR-486-3p and miR-486-5p) that were significantly altered in $\mathrm{PCa}$ patients.

Receiver operator characteristics (ROC) curves were constructed to explore the sensitivity and specificity of urinary exosomal miR-375, miR-451a, miR-486-3p and
Table 2 Expression of Significantly Deregulated miRNAs Between PCa Patients and Healthy Controls Determined by NGS Technology

\begin{tabular}{|c|c|c|c|c|}
\hline \multirow[t]{2}{*}{ miRNAs } & \multicolumn{2}{|c|}{ Expression Level } & \multirow{2}{*}{$\begin{array}{l}\text { Upl } \\
\text { Down }\end{array}$} & \multirow{2}{*}{$\begin{array}{l}\mid \log 2 \\
(F C) \mid\end{array}$} \\
\hline & $\begin{array}{l}\text { Healthy } \\
\text { Control }\end{array}$ & $\mathbf{P C a}$ & & \\
\hline hsa-miR-16-2-3p & 0.71 & 11.23 & Up & 3.99 \\
\hline hsa-miR-45 Ia & $|28.5|$ & 1054.92 & $U_{p}$ & 3.04 \\
\hline hsa-miR-486-3p & 27.74 & 657.95 & Up & 4.57 \\
\hline hsa-miR-486-5p & 67.21 & 2081.85 & Up & 4.95 \\
\hline hsa-miR-I26-5p & 0.00 & 4.24 & Up & 15.37 \\
\hline hsa-miR-1273g-3p & 7.74 & 43.44 & $U_{p}$ & 2.49 \\
\hline hsa-miR-199a-5p & 3.00 & 19.02 & $U_{p}$ & 2.66 \\
\hline hsa-miR-20a-5p & 5.24 & 26.20 & Up & 2.32 \\
\hline hsa-miR-3656 & 0.60 & 8.34 & $U_{p}$ & 3.80 \\
\hline hsa-miR-4492 & 5.39 & 38.21 & $U_{p}$ & 2.82 \\
\hline hsa-miR-4497 & 123.01 & 1018.97 & Up & 3.05 \\
\hline hsa-miR-4508 & 5.65 & 29.17 & $U_{p}$ & 2.37 \\
\hline hsa-miR-4532 & 8.54 & 43.11 & $U_{p}$ & 2.34 \\
\hline hsa-miR-9-5p & 20.11 & 84.12 & Up & 2.06 \\
\hline hsa-miR-375 & 839.54 & 36.23 & Down & 4.53 \\
\hline hsa-let-7c-5p & 103.31 & 6.90 & Down & 3.90 \\
\hline hsa-miR-100-5p & 2703.51 & 215.12 & Down & 3.65 \\
\hline hsa-miR-10a-5p & 3603.55 & 136.07 & Down & 4.73 \\
\hline hsa-miR-10b-3p & 9.33 & 0.00 & Down & 16.51 \\
\hline hsa-miR-I0b-5p & 1263.99 & 25.27 & Down & 5.64 \\
\hline hsa-miR-125a-5p & 143.39 & 18.04 & Down & 2.99 \\
\hline hsa-miR-125b-5p & 227.43 & 27.48 & Down & 3.05 \\
\hline hsa-miR-148a-3p & 1995.73 & 261.68 & Down & 2.93 \\
\hline hsa-miR-149-5p & 15.13 & 1.08 & Down & 3.80 \\
\hline hsa-miR-I5la-3p & 589.58 & 42.22 & Down & 3.80 \\
\hline hsa-miR-I52-3p & 78.38 & 4.43 & Down & 4.15 \\
\hline hsa-miR-192-5p & 1001.74 & 74.54 & Down & 3.75 \\
\hline hsa-miR-196a-5p & 16.69 & 1.05 & Down & 3.99 \\
\hline hsa-miR-200a-5p & 39.50 & 1.39 & Down & 4.83 \\
\hline hsa-miR-200b-3p & 381.33 & 22.83 & Down & 4.06 \\
\hline hsa-miR-200b-5p & 11.82 & 0.23 & Down & 5.70 \\
\hline hsa-miR-200c-3p & 348.95 & 21.77 & Down & 4.00 \\
\hline hsa-miR-203a-3p & 139.19 & 8.39 & Down & 4.05 \\
\hline hsa-miR-204-5p & 289.56 & 19.36 & Down & 3.90 \\
\hline hsa-miR-205-5p & 85.99 & 14.43 & Down & 2.58 \\
\hline hsa-miR-23b-3p & 30.03 & 3.50 & Down & 3.10 \\
\hline hsa-miR-27b-3p & 103.87 & 15.73 & Down & 2.72 \\
\hline hsa-miR-28-3p & 145.02 & 12.22 & Down & 3.57 \\
\hline hsa-miR-30a-3p & 276.16 & 12.15 & Down & 4.51 \\
\hline hsa-miR-30a-5p & 3273.13 & 104.62 & Down & 4.97 \\
\hline hsa-miR-30b-5p & 19.22 & 2.37 & Down & 3.02 \\
\hline hsa-miR-30c-5p & 160.87 & 17.00 & Down & 3.24 \\
\hline hsa-miR-30d-5p & 920.00 & 114.22 & Down & 3.01 \\
\hline hsa-miR-30e-3p & 9.54 & 0.85 & Down & 3.49 \\
\hline hsa-miR-36I-3p & 108.73 & 12.48 & Down & 3.12 \\
\hline hsa-miR-365a-5p & 3.86 & 0.00 & Down & 15.24 \\
\hline
\end{tabular}

(Continued) 
Table 2 (Continued).

\begin{tabular}{|l|l|l|l|l|}
\hline \multirow{2}{*}{ miRNAs } & \multicolumn{2}{|l|}{ Expression Level } & Up/ & |log2 \\
& $\begin{array}{l}\text { Healthy } \\
\text { Control }\end{array}$ & PCa & & \\
\cline { 2 - 3 } & (FC)| \\
\hline hsa-miR-429 & 13.27 & 1.40 & Down & 3.25 \\
hsa-miR-509-3p & 7.40 & 0.00 & Down & 16.18 \\
hsa-miR-5 14a-3p & 21.39 & 0.76 & Down & 4.82 \\
hsa-miR-532-5p & 294.17 & 24.93 & Down & 3.56 \\
hsa-miR-664a-5p & 12.81 & 0.88 & Down & 3.86 \\
hsa-miR-99b-3p & 21.03 & 0.84 & Down & 4.65 \\
hsa-miR-99b-5p & 490.76 & 30.43 & Down & 4.01 \\
\hline
\end{tabular}

miR-486-5p in discriminating between $\mathrm{PCa}$ patients and healthy controls. The results suggested that the four miRNAs can discriminate between the two groups with high precision: miR-375 (AUC $=0.788 ; 95 \% \mathrm{CI}=0.676-0.901)$, miR-451a $(\mathrm{AUC}=0.757 ; 95 \% \mathrm{CI}=0.641-0.872), \mathrm{miR}-486-3 \mathrm{p}(\mathrm{AUC}=$ $0.704 ; 95 \% \mathrm{CI}=0.576-0.833)$, miR-486-5p $(\mathrm{AUC}=0.796$; $95 \% \mathrm{CI}=0.690-0.901)$. As a result, we selected a classifier of four miRNAs to be the potential signature for PCa. The diagnostic sensitivity and specificity obtained for the validation cohort were $91 \%$ and $89 \%$, respectively, and AUC was 0.979 for the validation cohort (Figure 2).

\section{Correlation of Urinary Exosomal miRNA Levels with Clinical Factors}

To determine whether the four miRNA-based biomarkers were influenced by the clinical features of PCa patients, including age, Gleason score, T-stage and serum PSA, the relationships between the four urinary exosomal miRNA expression levels and clinical factors were analyzed using Spearman correlation. The urinary exosomal miR-375 level showed a correlation with the T-stage of $\mathrm{PCa}$ patients, while no obvious differences were observed when PCa patients were stratified by age, Gleason score, or serum PSA levels. In addition, the expression level of miR-375 was negatively correlated with the clinical T-stages of $\mathrm{PCa}, r=-0.41$. The PCa patients with lower levels of urinary exosomal miR-375 expression exhibited advanced clinical T-stages $(P<0.05)$.

\section{Differentiation Between PCa Patients and BPH Patients by a Biomarker Based on 2 miRNAs}

To investigate whether the four urinary exosomal miRNAs could serve as specific biomarkers to distinguish $\mathrm{PCa}$ from
$\mathrm{BPH}$, their expression levels in $29 \mathrm{BPH}$ patients were measured by using qRT-PCR, and the results were compared with those from the $\mathrm{PCa}$ patients in the validation cohort. The clinical and demographic characteristics of the PCa patients are summarized in Table 3. The results clearly indicated that miR-375 and miR-451a could be used to separate $\mathrm{PCa}$ from $\mathrm{BPH}$ cases (Figure 3A and B). No significant differences were observed in the levels of the other two candidate miRNAs between PCa patients and $\mathrm{BPH}$ patients (Figure $3 \mathrm{C}$ and $\mathrm{D}$ ). The ROC curve shows that the AUCs of miR-375 and miR-451a are 0.700 and 0.683 , respectively, which suggests their potential efficacy in distinguishing between $\mathrm{PCa}$ and $\mathrm{BPH}$. Moreover, miR-375 combined with miR-451a provided a better area under the curve $(\mathrm{AUC}=0.726 ; 95 \% \mathrm{CI}=$ 0.614-0.842), which means that the biomarker based on those 2 miRNAs is more sensitive in discriminating between PCa samples and BPH samples than PSA (AUC $=0.624 ; 95 \% \mathrm{CI}=0.487-0.761$; Figure 4 ).

\section{Differentiation Between Metastatic PCa Samples and Localized PCa Samples by miR-375}

To further analyse whether the 4 miRNAs could serve as a novel biomarker to predict the progression of $\mathrm{PCa}$, the $47 \mathrm{PCa}$ patients in the validation cohort were stratified into 2 groups: localized $\mathrm{PCa}$ and metastatic PCa. The comparison shown in Figure 5A clearly suggests that miR-375 could be used to distinguish localized $\mathrm{PCa}$ from metastatic $\mathrm{PCa}$. No significant differences were observed in the levels of the other three miRNAs between localized $\mathrm{PCa}$ and metastatic PCa (Figure 5BD). ROC analysis further confirmed that miR-375 (AUC $=0.806 ; 95 \% \mathrm{CI}=0.680-0.932)$ is superior to $\mathrm{PSA}$ $(\mathrm{AUC}=0.684 ; 95 \% \mathrm{CI}=0.510-0.859)$ in discriminating between localized $\mathrm{PCa}$ and metastatic $\mathrm{PCa}$ (Figure 6).

\section{Discussion}

$\mathrm{PCa}$ has a wide diffusion and represents the second major cause of death worldwide because of its aggressive behaviour. ${ }^{25}$ MiRNAs have been suggested to be novel diagnostic/therapeutic instruments, as they could play a role as a biomarker in the diagnosis and may also represent possible targets for new therapies. ${ }^{26,27}$ In the case of $\mathrm{PCa}$, the interest in miRNA is linked to the fact that prostate-specific antigen shows several 
A

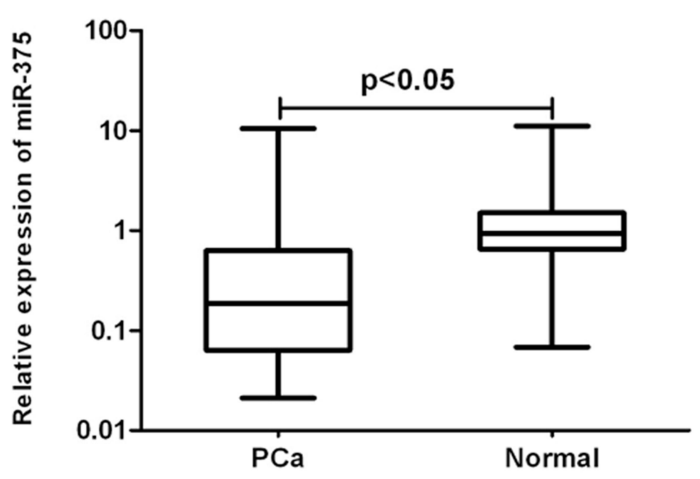

C

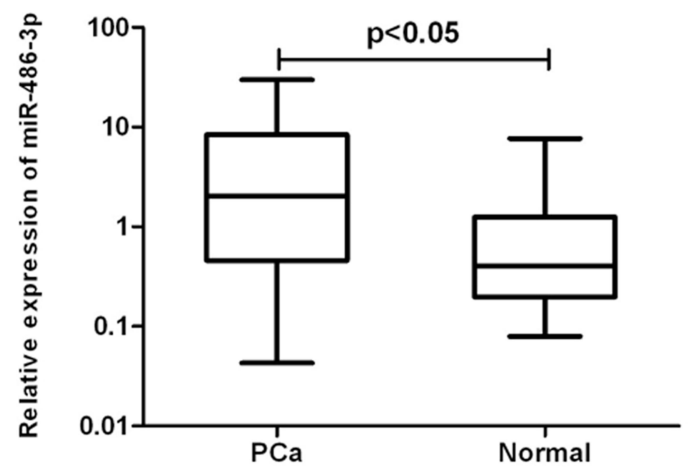

B

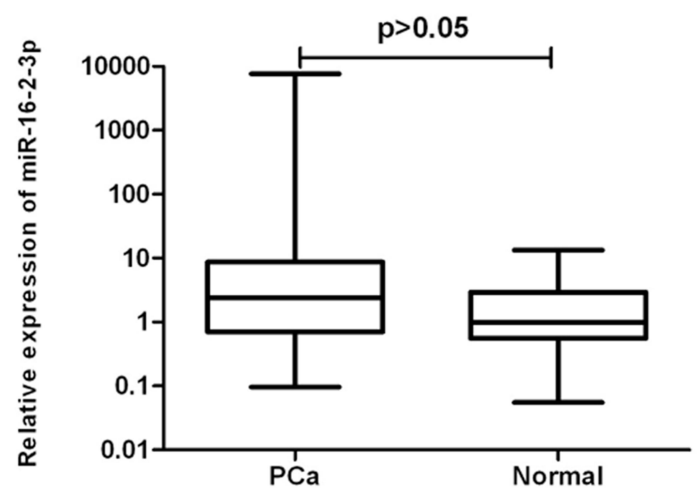

D

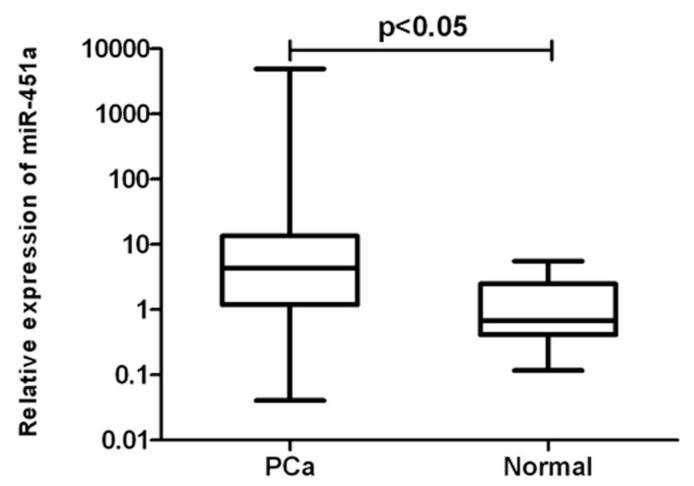

E

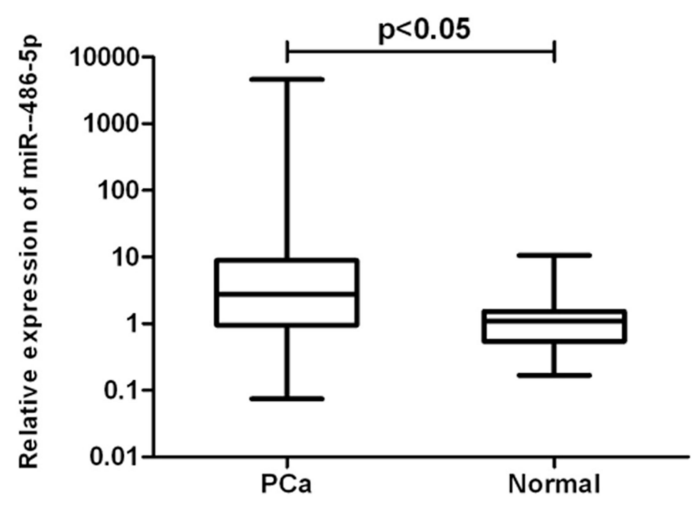

Figure I Expression of 5 candidate exosomal miRNAs in the urine samples of the validation cohort. Relative expression levels of urinary exosomal miR-375 (A), miR-I62-3p (B), miR-486-3p (C), miR-45 la (D), and miR-486-5p (E) in patients with PCa. Data presented as relative fold changes.

limitations, ${ }^{28,29}$ as infection, very common in frail patients. ${ }^{30,31}$ Early diagnosis, especially in the high risk, as the main cause of death for $\mathrm{PCa}$, is needed. ${ }^{32,33}$ The challenge nowadays is to better understand the mechanism of cancer development, finding real PCa markers and developing new specific therapies in order to reduce the mortality of PCa. ${ }^{34}$ Thus, the research on miRNA is of paramount importance. ${ }^{35}$ 


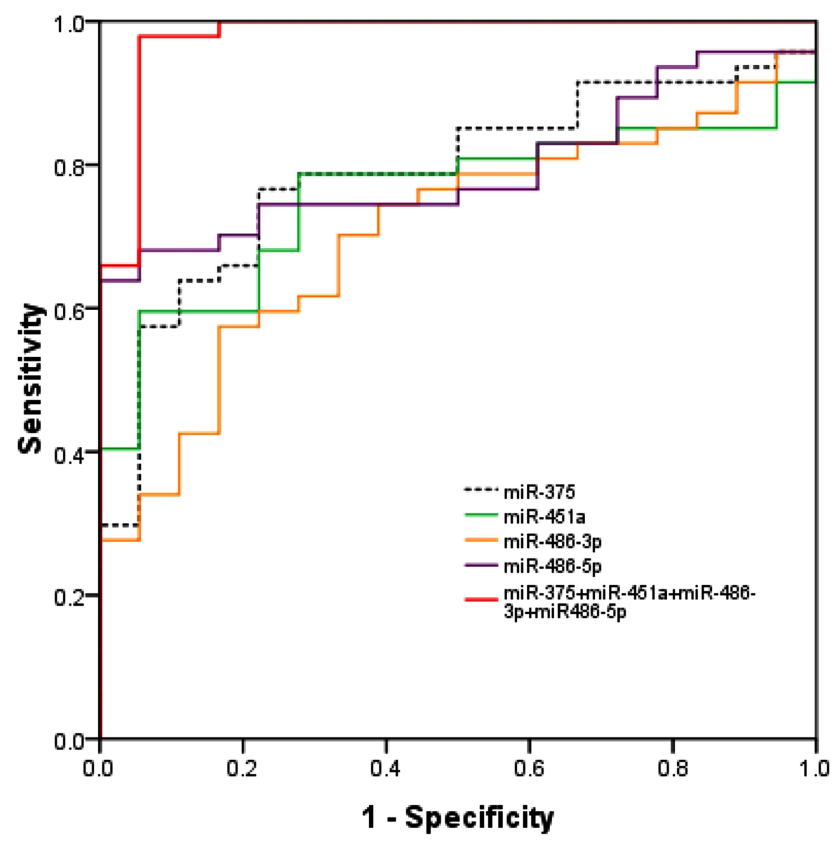

Figure 2 Receiver operator characteristic curve (ROC) for 4 individual miRNAs (miR-375, miR-45 Ia, miR-486-3p and miR-486-5p) and their combination to differentiate between PCa patients and healthy controls.

A large number of studies have proved that miRNAs play key roles in the pathogenesis and progression of tumours. $^{36,37}$ Therefore, miRNAs can be used as
Table 3 Clinical Characteristics of Participants in the qRT-PCR Cohort

\begin{tabular}{|c|c|c|c|}
\hline Characteristics & $\begin{array}{l}\text { PCa } \\
\text { Patients }\end{array}$ & $\begin{array}{l}\text { BPH } \\
\text { Patients }\end{array}$ & $\begin{array}{l}\text { Healthy } \\
\text { Controls }\end{array}$ \\
\hline $\begin{array}{l}\text { Total number } \\
\text { Age(years), } \\
\text { median (IQR) } \\
\text { PSA(ng/mL), } \\
\text { median (IQR) }\end{array}$ & $\begin{array}{l}47 \\
69.00 \\
(65.00-72.00) \\
16.91 \\
(7.42-48.76)\end{array}$ & $\begin{array}{l}29 \\
68.50 \\
(61.25-72.00) \\
2.77 \\
(1.35-6.97)\end{array}$ & $\begin{array}{l}25 \\
68.50 \\
(61.25-71.00) \\
0.93 \\
(0.64-1.68)\end{array}$ \\
\hline $\begin{array}{l}\text { Gleason score } \\
\quad \leq 6 \\
7 \\
\geq 8\end{array}$ & $\begin{array}{l}6 \\
19 \\
22\end{array}$ & & \\
\hline $\begin{array}{l}\text { Clinical stage } \\
\qquad \leq \mathrm{T} 2 \\
>\mathrm{T} 2\end{array}$ & $\begin{array}{l}26 \\
21\end{array}$ & & \\
\hline $\begin{array}{l}\text { Metastasis } \\
\text { No } \\
\text { Yes }\end{array}$ & $\begin{array}{l}32 \\
15\end{array}$ & & \\
\hline
\end{tabular}

molecular markers for diagnosis and prognosis. In general, using urine as a specimen for tumour biomarkers is challenging because urine contains a wide variety of biomolecules such as high concentrations of nucleases
A

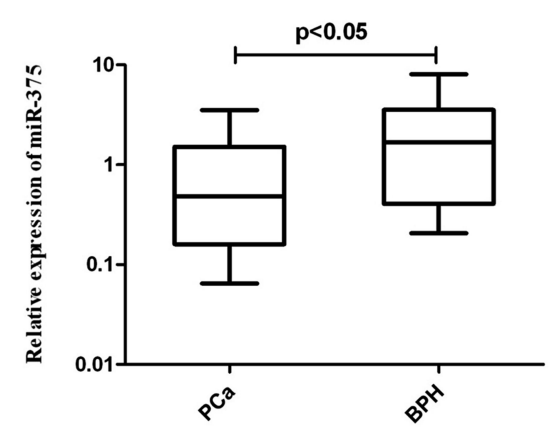

C

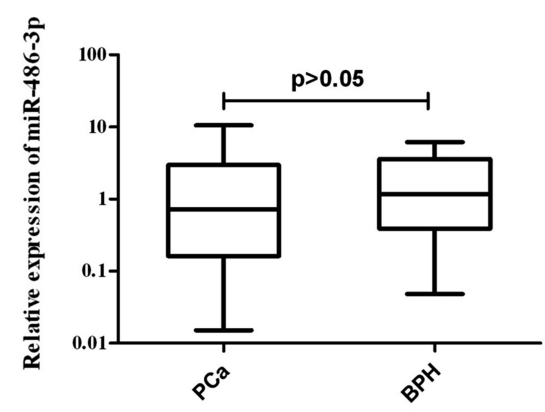

B

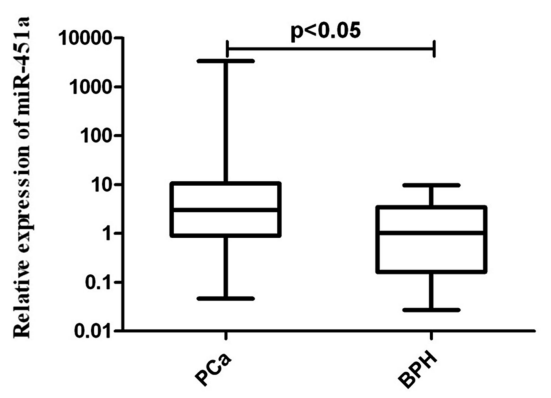

D

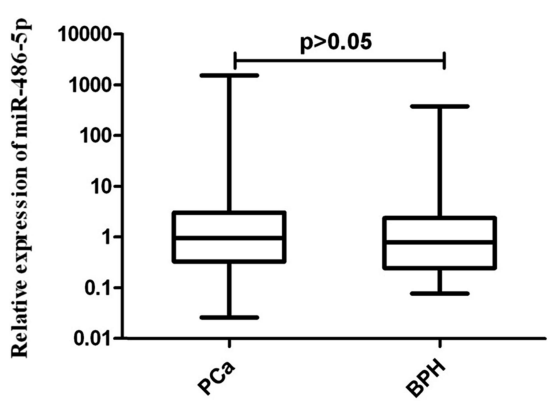

Figure 3 Expression of urinary exosomal miRNAs in PCa patients and BPH patients. Relative expression levels of urinary exosomal miR-375 (A), miR-45 Ia (B), miR-486-3P (C), and miR-486-5p (D). Data are presented as relative fold changes. 
and RNases. However, exosomes, which contain miRNA, have double-lipid membranes that can protect the miRNA contained within from degradation by RNases in urine. ${ }^{38}$ In addition, urine samples can be easily collected during the routine examination process. Based on the above advantages, urinary exosomal miRNAs can be used as promising molecular markers for certain diseases. Although miRNA levels have been studied in various diseases, few comprehensive studies have been reported so far for urine exosomal miRNAbased PCa diagnosis. ${ }^{39,40}$

The aim of this study was to explore whether urine samples contain exosomal miRNAs and whether the expression profiling of these urinary exosomal miRNAs consistently differ between PCa patients and healthy controls. In this study, we investigated hundreds of known exosomal miRNAs to search for diagnostic markers for $\mathrm{PCa}$ by using Illumina NGS technology to analyse the urine-based profile of exosomal miRNA expression. After analysis, we selected five significantly deregulated miRNAs, which showed a mean fold-change $>2$ between $\mathrm{PCa}$ patients and healthy controls, as well as between localized PCa and metastatic PCa. The 5 candidate miRNAs were further validated in a larger cohort including $47 \mathrm{PCa}$

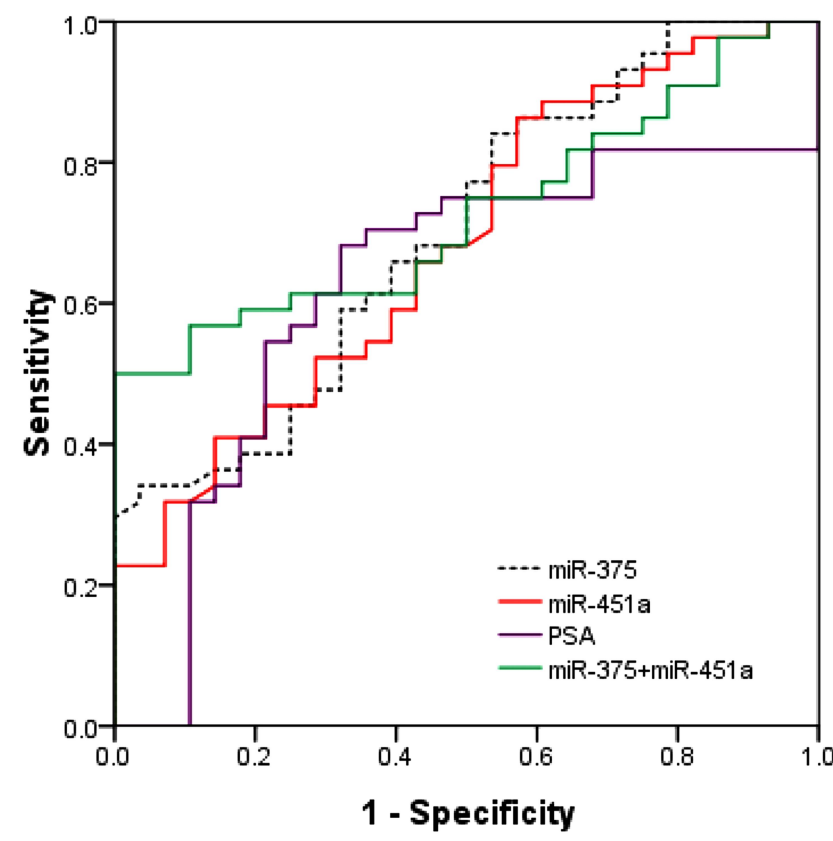

Figure 4 Evaluation of the ability of the miRNA-based panel and PSA to differentiate between patients with PCa and patients with BPH. Receiver operator characteristic (ROC) curve for PSA, two individual miRNAs (miR-375 and miR$45 \mathrm{I}$ a) and their combination to differentiate between two groups consisting of 47 $\mathrm{PCa}$ patients and $29 \mathrm{BPH}$ patients. patients and 25 healthy controls. qRT-PCR results demonstrated the upregulation of three miRNAs (miR451a, miR-486-3p, and miR-486-5p) and the downregulation of miR-375 in PCa samples. Furthermore, the expression level of miR-375 was found to be negatively correlated with advanced clinical T-stages of PCa patients.

In our research, we found that the exosomal miR-451a level, which has been shown to be frequently downregulated in hypopharyngeal squamous cell carcinoma, ${ }^{41}$ papillary thyroid carcinoma, ${ }^{42}$ melanoma $^{43}$ and gastric cancer, ${ }^{44}$ was significantly upregulated in the urine of PCa patients. Deregulation of miR-451a has not been previously reported in $\mathrm{PCa}$. To our knowledge, this is the first study to show that urinary exosomal miR-451a is aberrantly expressed in $\mathrm{PCa}$, which implies that miR451a could be a non-coding oncogene in $\mathrm{PCa}$, and its function needs further exploration. We found that urinary exosomal miR-486-5p and miR-486-3p levels were upregulated in PCa patients compared with healthy controls. To date, miR-486-5p and miR-486-3p have been rarely reported in $\mathrm{PCa}$ patients, ${ }^{45}$ which still needs further research.

For miR-375, previous studies showed its upregulation in PCa serum, PCa tissue and cell lines in comparison to healthy control serum, adjacent normal tissue and non-tumorigenic epithelial prostatic cells, respectively. ${ }^{46-49}$ Recently, Foj et al detected miR-375 in urinary exosomes and found it was significantly upregulated in PCa patients. ${ }^{39}$ However, in the present study based on NGS and qRT-PCR studies, we found an opposite result. Interestingly, in another recent study, the authors found a similar pattern. They indicated that the levels of urinary exosomal miR-196a-5p and miR501-3p, which had been found to be mainly upregulated in $\mathrm{PCa}$ patients in previous studies, were significantly downregulated in PCa patients based on both NGS and qRT-PCR tests. While in our present NGS study, we achieved consistent results showing the downregulation of the two miRNAs in PCa patients. In addition, in the above-mentioned study, the entire list of NGS results showed that miR-375 was reduced in PCa patients compared with the healthy controls, which agreed with our results. It is not clear why there are such different results in urinary exosomal miRNAs studies, but the different patient cohorts using could be a possible explanation. Therefore, to confirm the findings, larger cohorts and more multi-centre studies are needed. In addition, 
A

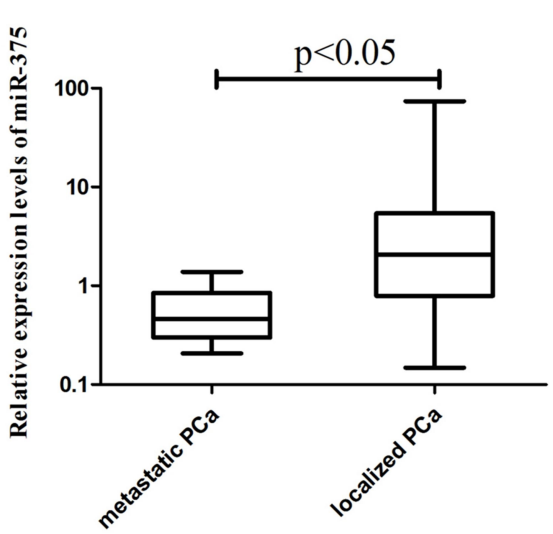

C

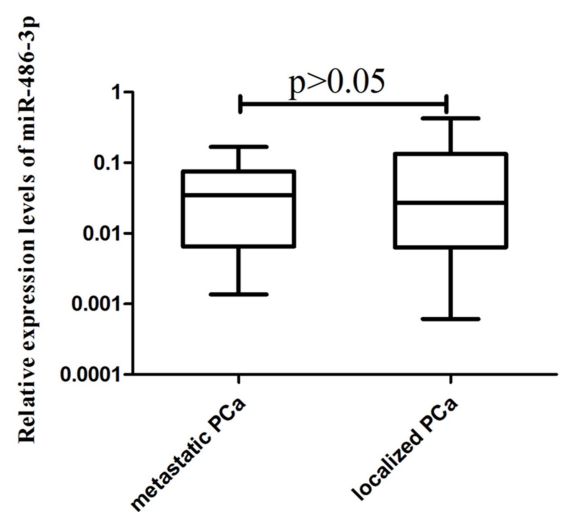

B

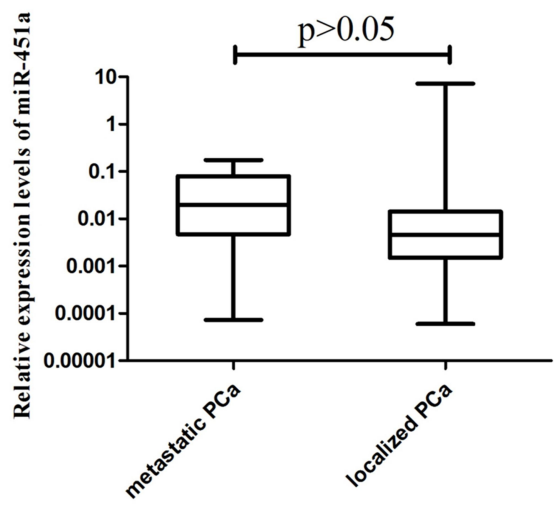

D

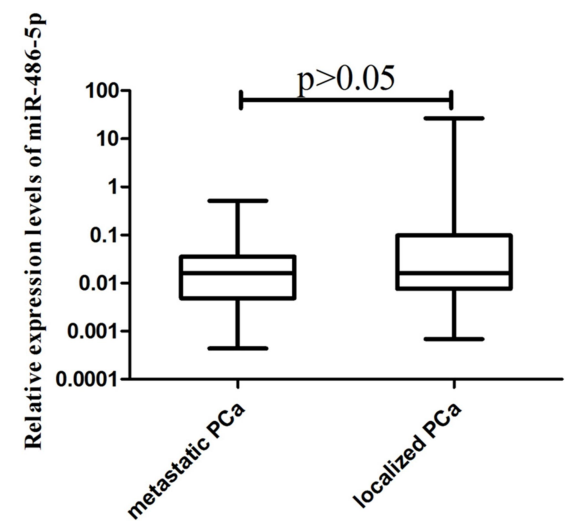

Figure 5 Expression of urinary exosomal miRNAs in localized PCa and metastatic PCa. Relative expression levels of urinary exosomal miR-375 (A), miR-45 Ia (B), miR-486$3 \mathrm{P}(\mathbf{C})$, and miR-486-5p (D) in localized PCa patients and metastatic PCa patients. Data presented as relative fold changes.

although miR-375 has been mostly considered to be a tumour oncogene in $\mathrm{PCa}$, it is worth mentioning that the recent literature has revealed that miR-375 acts either as an oncomiR or a tumour-suppressor miRNA depending on the cellular context, which seems to agree with the results in our study.

This study has demonstrated the presence of exosomal miRNAs in urine samples as in previous reports. In addition, we have demonstrated that exosomal miR-375, miR451a, miR-486-3p and miR-486-5p are differentially expressed in urine from PCa patients, and the ROC curve demonstrates that the four miRNAs combined together have good efficacy to distinguish between healthy controls and $\mathrm{PCa}$ patients, with a sensitivity of $91 \%$ and a specificity of $89 \%$. In addition, our present finding suggests that a combination of miR-375 and miR-451a has the potential to serve as a specific and sensitive molecular marker to distinguish $\mathrm{PCa}$ patients from BPH patients, and miR-375 alone can distinguish localized $\mathrm{PCa}$ patients

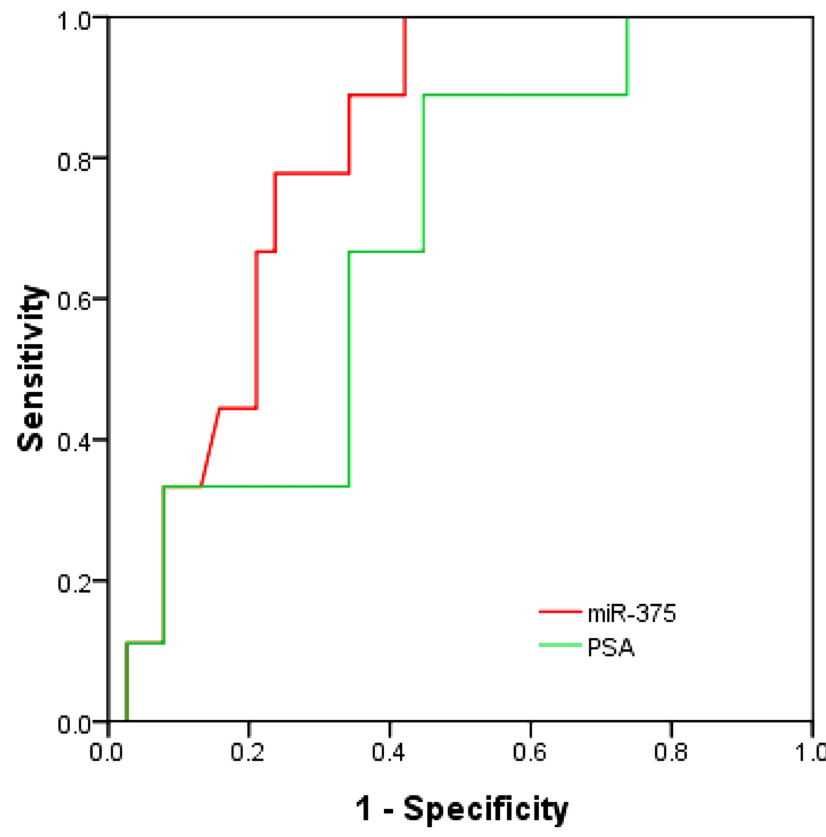

Figure 6 Receiver-operating characteristic curve (ROC) for urinary exosomal miR-375 and PSA to differentiate between localized PCa patients and metastatic PCa patients. 
from metastatic $\mathrm{PCa}$ patients to predict the progression of $\mathrm{PCa}$.

\section{Conclusion}

In conclusion, we suggest that certain exosomal miRNAs in urine could serve as powerful and non-invasive tools for the diagnosis and prognosis of PCa. Further studies are needed in a larger cohort of patients to confirm these findings.

\section{Abbreviations}

AUC, area under the curve; $\mathrm{Ct}$, threshold cycle; miRNAs, microRNAs; NGS, next-generation sequencing; PCa, prostate cancer; PSA, prostate-specific antigen; ROC, receiveroperating characteristic; TEM, transmission electron microscopy.

\section{Data Sharing Statement}

All the datasets in the manuscript are available from the corresponding author on request.

\section{Ethics Approval and Consent to Participate}

The procedures were approved by the Ethics Committee of Xijing Hospital, and the written informed consent was signed by each patient.

\section{Acknowledgments}

This work was supported by grants from the National Natural Science Foundation of China (General Program, 81872347), the Health Commission of Shaanxi Province (2016D020), the Xi'an Science and Technology Bureau (2017121SF/YX015(3)) and Shaanxi Natural Science Foundation (2018JQ8010).

\section{Author Contributions}

All authors contributed to data analysis, drafting or revising the article, have agreed on the journal to which the article will be submitted, gave final approval of the version to be published, and agree to be accountable for all aspects of the work.

\section{Disclosure}

The authors declare that no competing interests exist.

\section{References}

1. Siegel RL, Miller KD, Jemal A. Cancer statistics, 2017. CA Cancer J Clin. 2017;67(1):7-30. doi:10.3322/caac.21387
2. Grossman DC, Curry SJ, Owens DK, et al. Screening for prostate cancer: US preventive services task force recommendation statement. JAMA. 2018;319(18):1901-1913. doi:10.1001/jama.2018.3710

3. Lilja H, Ulmert D, Vickers AJ. Prostate-specific antigen and prostate cancer: prediction, detection and monitoring. Nat Rev Cancer. 2008;8 (4):268-278. doi:10.1038/nrc2351

4. Neylon A, McGorrian C, Blake GJ. miRNA-93-5p and other miRNAs as predictors of coronary artery disease and STEMI. Int J Cardiol. 2016;224:310-316. doi:10.1016/j.ijcard.2016.09.016

5. Giallombardo M, Chacártegui Borrás J, Castiglia M, et al. Exosomal miRNA analysis in non-small cell lung cancer (NSCLC) patients' plasma through qPCR: a feasible liquid biopsy tool. JoVE. 2016; (111). doi: $10.3791 / 53900$

6. Delić D, Eisele C, Schmid R, et al. Urinary exosomal miRNA signature in type II diabetic nephropathy patients. PLoS One. 2016;11(3):e0150154. doi:10.1371/journal.pone.0150154

7. Shi R, Wang PY, Li XY, et al. Exosomal levels of miRNA-21 from cerebrospinal fluids associated with poor prognosis and tumor recurrence of glioma patients. Oncotarget. 2015;6(29):26971-26981. doi:10.18632/oncotarget.4699

8. Cappello F, Logozzi M, Campanella C, et al. Exosome levels in human body fluids: a tumor marker by themselves? Eur J Pharm Sci. 2017;96:93-98. doi:10.1016/j.ejps.2016.09.010

9. Zhang J, Li S, Li L, et al. Exosome and exosomal microRNA: trafficking, sorting, and function. Genomics Proteomics Bioinformatics. 2015;13(1):17-24. doi:10.1016/j.gpb.2015.02.001

10. Colombo M, Raposo G, Théry C. Biogenesis, secretion, and intercellular interactions of exosomes and other extracellular vesicles. Annu Rev Cell Dev Biol. 2014;30:255-289. doi:10.1146/annurevcellbio-101512-122326

11. Franzen CA, Blackwell RH, Foreman KE, Kuo PC, Flanigan RC, Gupta GN. Urinary exosomes: the potential for biomarker utility, intercellular signaling and therapeutics in urological malignancy. J Urol. 2016;195(5):1331-1339. doi:10.1016/j.juro.2015.08.115

12. Iwai K, Minamisawa T, Suga K, Yajima Y, Shiba K. Isolation of human salivary extracellular vesicles by iodixanol density gradient ultracentrifugation and their characterizations. $J$ Extracell Vesicles. 2016;5:30829. doi:10.3402/jev.v5.30829

13. Skog J, Würdinger T, van Rijn S, et al. Glioblastoma microvesicles transport RNA and proteins that promote tumour growth and provide diagnostic biomarkers. Nat Cell Biol. 2008;10(12):1470-1476. doi: $10.1038 /$ ncb1800

14. Cheng L, Sun X, Scicluna BJ, Coleman BM, Hill AF. Characterization and deep sequencing analysis of exosomal and non-exosomal miRNA in human urine. Kidney Int. 2014;86 (2):433-444. doi:10.1038/ki.2013.502

15. Gildea JJ, Carlson JM, Schoeffel CD, Carey RM, Felder RA. Urinary exosome miRNome analysis and its applications to salt sensitivity of blood pressure. Clin Biochem. 2013;46(12):1131-1134. doi:10.1016/ j.clinbiochem.2013.05.052

16. Huang X, Yuan T, Liang M, et al. Exosomal miR-1290 and miR-375 as prognostic markers in castration-resistant prostate cancer. Eur Urol. 2015;67(1):33-41. doi:10.1016/j.eururo.2014.07.035

17. Haflidadóttir BS, Ceder Y. Exosomal microRNAs as potential biomarkers in castration-resistant prostate cancer. Eur Urol. 2015;67 (1):42-43. doi:10.1016/j.eururo.2014.08.067

18. Ogata-Kawata H, Izumiya M, Kurioka D, et al. Circulating exosomal microRNAs as biomarkers of colon cancer. PLoS One. 2014;9(4): e92921. doi:10.1371/journal.pone.0092921

19. Corcoran C, Rani S, O'Driscoll L. miR-34a is an intracellular and exosomal predictive biomarker for response to docetaxel with clinical relevance to prostate cancer progression. Prostate. 2014;74 (13):1320-1334. doi:10.1002/pros.22848

20. Hessvik NP, Sandvig K, Llorente A. Exosomal miRNAs as biomarkers for prostate cancer. Front Genet. 2013;4:36. doi:10.3389/ fgene.2013.00036 
21. Li Z, Ma YY, Wang J, et al. Exosomal microRNA-141 is upregulated in the serum of prostate cancer patients. Onco Targets Ther. 2016;9:139-148. doi:10.2147/OTT.S95565

22. Selth LA, Townley S, Gillis JL, et al. Discovery of circulating microRNAs associated with human prostate cancer using a mouse model of disease. Int J Cancer. 2012;131(3):652-661. doi:10.1002/ijc.26405

23. Mitchell PS, Parkin RK, Kroh EM, et al. Circulating microRNAs as stable blood-based markers for cancer detection. Proc Natl Acad Sci USA. 2008;105(30):10513-10518. doi:10.1073/pnas.0804549105

24. Livak KJ, Schmittgen TD. Analysis of relative gene expression data using real-time quantitative PCR and the 2(-Delta Delta C(T)) method. Methods (San Diego, Calif). 2001;25(4):402-408. doi:10.10 06/meth.2001.1262

25. Situ J, Zhang H, Jin Z, Li K, Mao Y, Huang W. MicroRNA-939 directly targets HDGF to inhibit the aggressiveness of prostate cancer via deactivation of the WNT/ $\beta$-catenin pathway. Onco Targets Ther. 2020;13:4257-4270. doi:10.2147/OTT.S250101

26. Cochetti G, Poli G, Guelfi G, Boni A, Egidi MG, Mearini E. Different levels of serum microRNAs in prostate cancer and benign prostatic hyperplasia: evaluation of potential diagnostic and prognostic role. Onco Targets Ther. 2016;9:7545-7553. doi:10.2147/OTT.S119027

27. Guelfi G, Cochetti G, Stefanetti V, et al. Next generation sequencing of urine exfoliated cells: an approach of prostate cancer microRNAs research. Sci Rep. 2018;8(1):7111. doi:10.1038/s41598-018-24236-y

28. Mearini E, Antognelli C, Del Buono C, et al. The combination of urine DD3(PCA3) mRNA and PSA mRNA as molecular markers of prostate cancer. Biomarkers. 2009;14(4):235-243. doi:10.1080/13547 500902807306

29. Talesa VN, Antognelli C, Del Buono C, et al. Diagnostic potential in prostate cancer of a panel of urinary molecular tumor markers. Cancer Biomarkers. 2009;5(6):241-251. doi:10.3233/CBM-20090109

30. Errico G, Gagliotti C, Monaco M, et al. Colonization and infection due to carbapenemase-producing Enterobacteriaceae in liver and lung transplant recipients and donor-derived transmission: a prospective cohort study conducted in Italy. Clin Microbiol Infect. 2019;25 (2):203-209. doi:10.1016/j.cmi.2018.05.003

31. Gagliotti C, Morsillo F, Moro ML, et al. Infections in liver and lung transplant recipients: a national prospective cohort. Eur J Clin Microbiol Infect Dis. 2018;37(3):399-407. doi:10.1007/s10096-018-3183-0

32. Egidi MG, Cochetti G, Guelfi G, et al. Stability assessment of candidate reference genes in urine sediment of prostate cancer patients for miRNA applications. Dis Markers. 2015;2015:973597. doi: $10.1155 / 2015 / 973597$

33. Rende M, Rambotti MG, Stabile AM, et al. Novel localization of low affinity NGF receptor (p75) in the stroma of prostate cancer and possible implication in neoplastic invasion: an immunohistochemical and ultracytochemical study. Prostate. 2010;70(5):555-561. doi:10.1002/pros.21089

34. Baldassarri M, Fallerini C, Cetta F, et al. Omic approach in non-smoker female with lung squamous cell carcinoma pinpoints to germline susceptibility and personalized medicine. Cancer Research Treat. 2018;50(2):356-365. doi:10.4143/crt.2017.125
35. Cochetti G, Rossi de Vermandois JA, Maulà V, et al. Role of miRNAs in prostate cancer: do we really know everything? Urol Oncol. 2020;38(7):623-635. doi:10.1016/j.urolonc.2020.03.007

36. Hayes J, Peruzzi PP, Lawler S. MicroRNAs in cancer: biomarkers, functions and therapy. Trends Mol Med. 2014;20(8):460-469. doi:10.1016/j.molmed.2014.06.005

37. Cho WC. OncomiRs: the discovery and progress of microRNAs in cancers. Mol Cancer. 2007;6:60. doi:10.1186/1476-4598-6-60

38. Zhang W, Peng P, Shen K. Role of exosome shuttle RNA in cell-tocell communication. Zhongguo Yi Xие Ke Xие Yиаn Хие Bao. 2016;38(4):480-483. doi:10.3881/j.issn.1000-503X.2016.04.020

39. Foj L, Ferrer F, Serra M, et al. Exosomal and non-exosomal urinary miRNAs in prostate cancer detection and prognosis. Prostate. 2017;77(6):573-583. doi:10.1002/pros.23295

40. Filella X, Foj L. Prostate cancer detection and prognosis: from prostate specific antigen (PSA) to exosomal biomarkers. Int $\mathrm{J} \mathrm{Mol}$ Sci. 2016;17(11):1784. doi:10.3390/ijms17111784

41. Fukumoto I, Kinoshita T, Hanazawa T, et al. Identification of tumour suppressive microRNA-451a in hypopharyngeal squamous cell carcinoma based on microRNA expression signature. $\mathrm{Br} J$ Cancer. 2014;111(2):386-394. doi:10.1038/bjc.2014.293

42. Li M, Song Q, Li H, Lou Y, Wang L. Circulating miR-25-3p and miR-451a may be potential biomarkers for the diagnosis of papillary thyroid carcinoma. PLoS One. 2015;10(7):e0132403. doi:10.1371/ journal.pone. 0132403

43. Babapoor S, Fleming E, Wu R, Dadras SS. A novel miR-451a isomiR, associated with amelanotypic phenotype, acts as a tumor suppressor in melanoma by retarding cell migration and invasion. PLoS One. 2014;9(9):e107502. doi:10.1371/journal.pone.0107502

44. Riquelme I, Tapia O, Leal P, et al. miR-101-2, miR-125b-2 and miR-451a act as potential tumor suppressors in gastric cancer through regulation of the $\mathrm{PI} 3 \mathrm{~K} / \mathrm{AKT} / \mathrm{mTOR}$ pathway. Cell Oncol (Dordrecht). 2016;39(1):23-33. doi:10.1007/s13402-015-0247-3

45. Yang Y, Ji C, Guo S, et al. The miR-486-5p plays a causative role in prostate cancer through negative regulation of multiple tumor suppressor pathways. Oncotarget. 2017;8(42):72835-72846. doi:10.18 632/oncotarget. 20427

46. Pickl JM, Tichy D, Kuryshev VY, et al. Ago-RIP-Seq identifies Polycomb repressive complex I member CBX7 as a major target of miR-375 in prostate cancer progression. Oncotarget. 2016;7 (37):59589-59603. doi:10.18632/oncotarget.10729

47. Wach S, Al-Janabi O, Weigelt K, et al. The combined serum levels of miR-375 and urokinase plasminogen activator receptor are suggested as diagnostic and prognostic biomarkers in prostate cancer. Int J Cancer. 2015;137(6):1406-1416. doi:10.1002/ijc.29505

48. Choi N, Park J, Lee JS, et al. miR-93/miR-106b/miR-375-CICCRABP1: a novel regulatory axis in prostate cancer progression. Oncotarget. 2015;6(27):23533-23547. doi:10.18632/oncotarget.4372

49. Brase JC, Johannes M, Schlomm T, et al. Circulating miRNAs are correlated with tumor progression in prostate cancer. Int $J$ Cancer. 2011;128(3):608-616. doi:10.1002/ijc.25376
Cancer Management and Research is an international, peer-reviewed open access journal focusing on cancer research and the optimal use of preventative and integrated treatment interventions to achieve improved outcomes, enhanced survival and quality of life for the cancer patient.
The manuscript management system is completely online and includes a very quick and fair peer-review system, which is all easy to use. Visit http://www.dovepress.com/testimonials.php to read real quotes from published authors. 